\title{
Axanthism in amphibians: A review and the first record in the wide- spread toad of the Bufotes viridis complex (Anura: Bufonidae)
}

\author{
Daniel Jablonski ${ }^{1, *}$, Andrej Alena ${ }^{2}$, Petr Vlček ${ }^{3}$ \& David Jandzik ${ }^{1,4}$
}

\author{
1 Department of Zoology, Faculty of Natural Sciences, Comenius University in Bratislava, Mlynská dolina B-1, 84215 \\ Bratislava, Slovakia. \\ 2 Muškátová 16, 90201 Pezinok, Slovakia. \\ 3 Slovanská 5, 73601 Havíŕov-Město, Czech Republic. \\ 4 Department of Ecology and Evolutionary Biology (EBIO), Univeristy of Colorado at Boulder, Ramaley N122, Campus \\ Box 334, 80309-0334 Boulder, CO, USA. \\ * Corresponding author: daniel.jablonski@balcanica.cz
}

\begin{abstract}
Axanthism in amphibians is a relatively rare color aberration reported less often than leucism or albinism. It is caused by lack of specific types of pigment cells in the skin, namely xanthophores, erythrophores, and iridophores. Here, we present the first case of occurrence of this aberration in a widely distributed toad of the green toad (Bufotes viridis) complex and provide an extensive review of axanthism occurrence in amphibians. So far it has been reported in more than 20 species from nine families of amphibians with the highest occurrence in the family Ranidae.
\end{abstract}

KEY WORDS: amphibia, Bufo viridis, color aberration, Slovakia.

\section{INTRODUCTION}

Coloration plays an important role in various aspects of animal life history. In many species of amphibians and reptiles cryptic or aposematic coloration enhances protection against visual predators, while at the same time it can provide valuable information to cognates about sex, fitness, maturity or availability for reproduction (VITT \& CALDWELL, 2009). Therefore natural selection usually eliminates any aberrations that occur (ANDRÉN \& NILSON, 1981). However, there are several examples when specimens or even populations that are characterized by aberrant coloration, can survive and reproduce, e.g. melanic specimens of the common lizard Zootoca vivipara or an albinotic population of the snake Elaphe climacophora (SCHULZ, 1996; GVOŽDÍK, 1999; JAMBRICH \& JANDZIK, 2012). Changes in coloration are also often related to life in the dark, e.g. in caves, as is known in the Mexican tetra Astyanax mexicanus (MITCHELL et al., 1977; WILKENS, 1988), whose surface populations are normally pigmented, while the cave populations lack pigmentation. Another such example is the olm Proteus anguinus, a troglobiont urodele known in two forms: either completely unpigmented or with black pigmentation (SKET \& ARNTZEN, 1994).

Color aberrations that have been described in vertebrates are albinism, amelanism, axanthism, erythrism, hypomelanism, leucism, melanism and piebaldism, though this list is not exhaustive and nomenclature is not consensual (BECHTEL, 1995). In wild amphibians the literature reports albinism (white or yellowish body color and red eyes) and leucism (white or pinkish body color, dark eyes) as the most common forms of aberrant coloration (e.g. WERNER, 1893; SMALLCOMBE, 1949; CAMPANNA, 1973; DYRKACZ, 1981; BECHTEL, 1995; MiKULÍČEK et al., 2001; THOMAS ET AL., 2002; PABIJAN et al., 2004; LÓPEZ \& GHIRARDI, 2011; ESCORIZA, 2012). They usually result from gene mutations affecting development and distribution of 
chromatophores and/or skin pigment production (Duellman \& Trueb, 1994; Bechtel, 1995).

Axanthism characterized by blue, bluish or generally grey, dark body color, very dark patterns, and dark eyes, is one of the least known aberrations, though it is presumably as widespread as albinism (BECHTEL, 1995). Skin of axanthic animals lacks xanthophores (BROWDER, 1968), erythrophores, and iridophores, which normally produce yellow and orange, red, and lightreflecting and scattering pigments, respectively. In some cases, the xanthophores might be unable to produce the pigment, despite the fact they are present (BERNS \& NARAYAN, 1970). The lack of iridophores typically results in duller and darker overall coloration. In contrast to the skin, the eyes of axanthic specimens may contain iridophores and melanophores (rarely also xanthophores; BECHTEL, 1995; Frost-MASON \& MASON, 1996). So far, axanthism has been reported either in a partial or a complete form in several species of amphibians (e.g. LIU, 1931; DUBOIS \& VACHARD, 1971; DUBOIS, 1979; JUSZCZYK, 1987; BECHTEL, 1995; VLČEK, 2003, 2008; NiCCOLI, 2013; for other references and detailed information see Table 1).

\section{AXANTHISM IN THE GREEN TOAD BUFOTES VIRIDIS}

The taxonomically complicated species complex of green toads [Bufotes viridis (Laurenti, 1768), complex; also known as Bufo viridis in traditional taxonomy] includes at least 14 morphologically similar species distributed across Europe, Asia and Northern Africa (STÖCK et al., 2006, 2008; Frost, 2014). Coloration and pattern of these toads varies across their distribution range, but generally the background coloration is light to dark brown, covered with darker green patches creating a camouflage pattern. Tiny red spots might be present on various parts of the body. The belly is usually pale without a pattern and the iris is yellow or yellowish (ARNOLD \& OVENDEN, 2002). Interestingly, despite the variation in tint or intensity of both background color and pattern, color aberrations are relatively rare within this species complex. To the best of our knowledge, only albinism (FLINDT, 1985; ANDRÄ, 2011), erythrism (LANZA \& CANESTRELLI, 2002) and retinal depigmentation (ENGELMANN \& OBST, 1976) have been reported so far.

Here, we report an axanthic juvenile specimen (approx. SVL $30 \mathrm{~mm}$ ) of $B$. viridis, encountered in Pezinok, SW Slovakia $\left(48.31993^{\circ}\right.$ N $17.24085^{\circ}$ E; June 28, 2009). The specimen was overall very dark with some bluish tint on the belly. Brighter parts were confined to the area around mouth, throat and eyes and some brighter spots were also observed on parotid glands, dorsum, and hind limbs. Skin patches that normally form a camouflage pattern were also darker, though the contrast between them and the dark background was hardly detectable. Some poison gland openings had white or yellow tips, and tiny yellowish spots were also present on flanks anterior to hind limbs (Fig. 1). Between 35 and 50 normally pigmented specimens of $B$. viridis were observed in the area at the same time. From the total number of about 150 observed specimens in the season of 2009 , only the one reported here was axanthic $(0.7 \%)$.

\section{AXANTHISM IN AMPHIBIANS}

Axanthism remains a rarely observed and reported color aberration in amphibians when compared to other color aberrations (see DuBOIS, 1979; BECHTEL, 1995). In partially axanthic specimens it is easy to overlook this aberration, and completely axanthic specimens can easily be confused with melanic specimens. These, similarly to the axanthic ones, are also dark and have a somewhat disrupted pattern. Distinction between melanism and axanthism is possible based on the slightly lighter coloration of axanthic specimens, in which also the pattern is typically discernible. This is also the case of the green toad specimen reported herein. The anatomical explanation has been offered from studies on axolotls, in which melanic specimens 
lack both xanthophores and iridophores in their skin, but unlike axanthic specimens, they also over-proliferate melanophores resulting in much darker coloration (FROST-MASON \& MASON, 1996). According to our summarized literature data (see Table 1), three basic types of axanthism can be recognized in amphibians: (i) complete or partial blue body coloration (mainly in Ranidae, see Table 1 and references therein), (ii) complete or partial grayish or dark body coloration, (iii) normal body coloration with black eyes. These three types are not mutually exclusive and combinations can occur (e.g. blue coloration with black eyes).

\section{CAUSES OF AXANTHISM}

Although the genetic background of pigmentcell formation in amphibians is relatively well known (NEVO, 1973; Frost-MASON \& MASON, 1996; HOFFMAN \& BLOUIN, 2000), proximate causes of axanthism remain unknown. Besides genetic mechanisms, environmental causes cannot be excluded, such as temperature fluctuations, food quality, parasitism or environmental pollution (cf. Dubois, 1979; Vershinin, 2004; CABALlero et al., 2012). Interestingly, many observations of axanthic specimens (mainly in the family Ranidae; see Table 1) were reported from 19601990s from urban areas of industrial countries with potentially high levels of environmental pollution (cf. DANDOVÁ et al., 1995). However, in some cases the darker coloration can offer an adaptive advantage, e.g. in thermoregulation, which is particularly important in ectothermic vertebrates (e.g. melanic Vipera berus; ANDRÉN $\&$ NiLSON, 1981). Conversely, loss of cryptic or aposematic pattern and coloration might lead to an increased conspicuousness and decreased intensity of warning signals, respectively, and thus a higher predation risk (CHILDS, 1953; ANDRÉN \& NiLSON, 1981). It is possible that in axanthic specimens a genetic relationship exists between
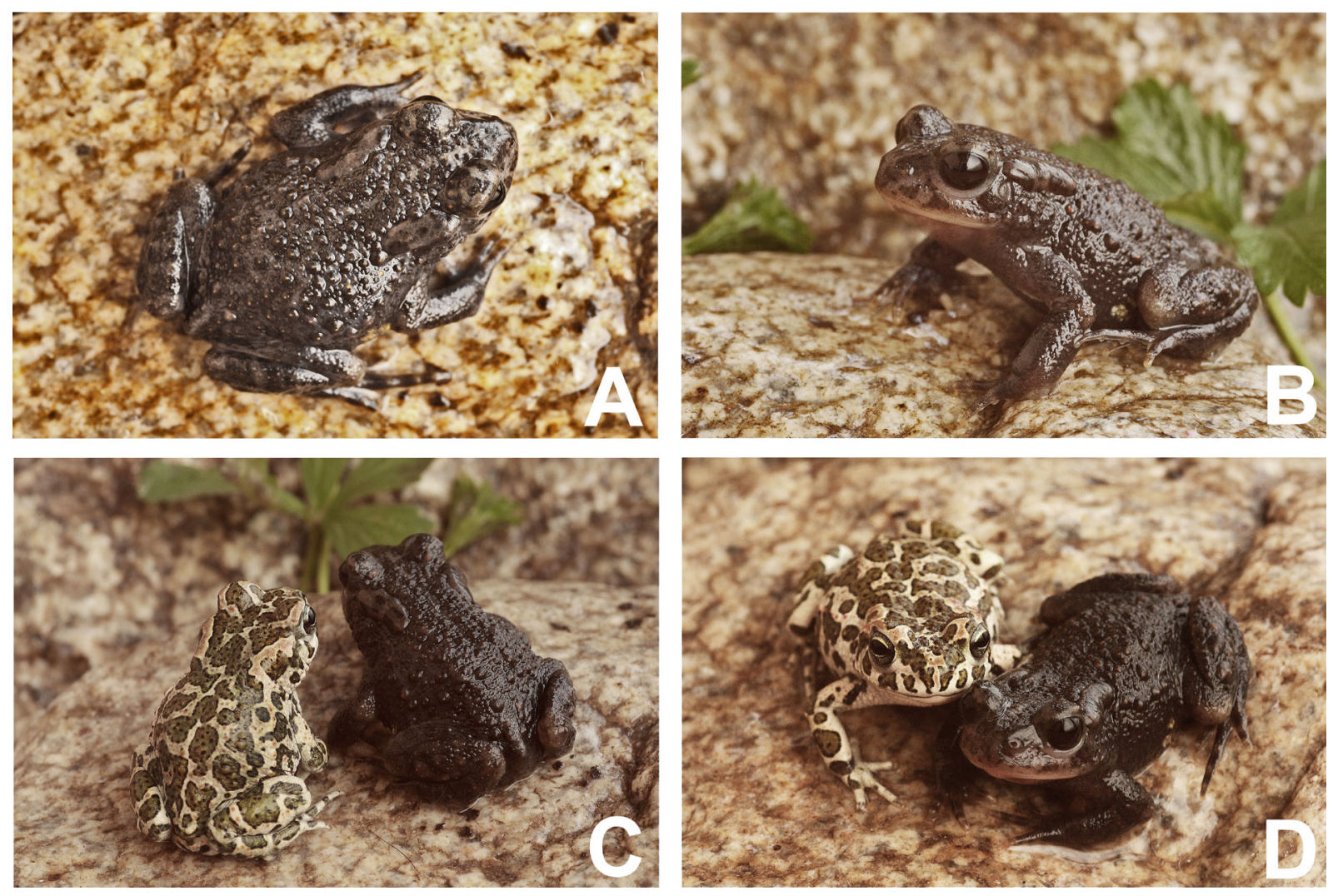

Fig. 1 - Axanthic specimen of the green toad Bufotes viridis from Slovakia. A: dorsal view; B: lateral view; C-D: comparison with normally colored specimen of the same size and from the same locality. 


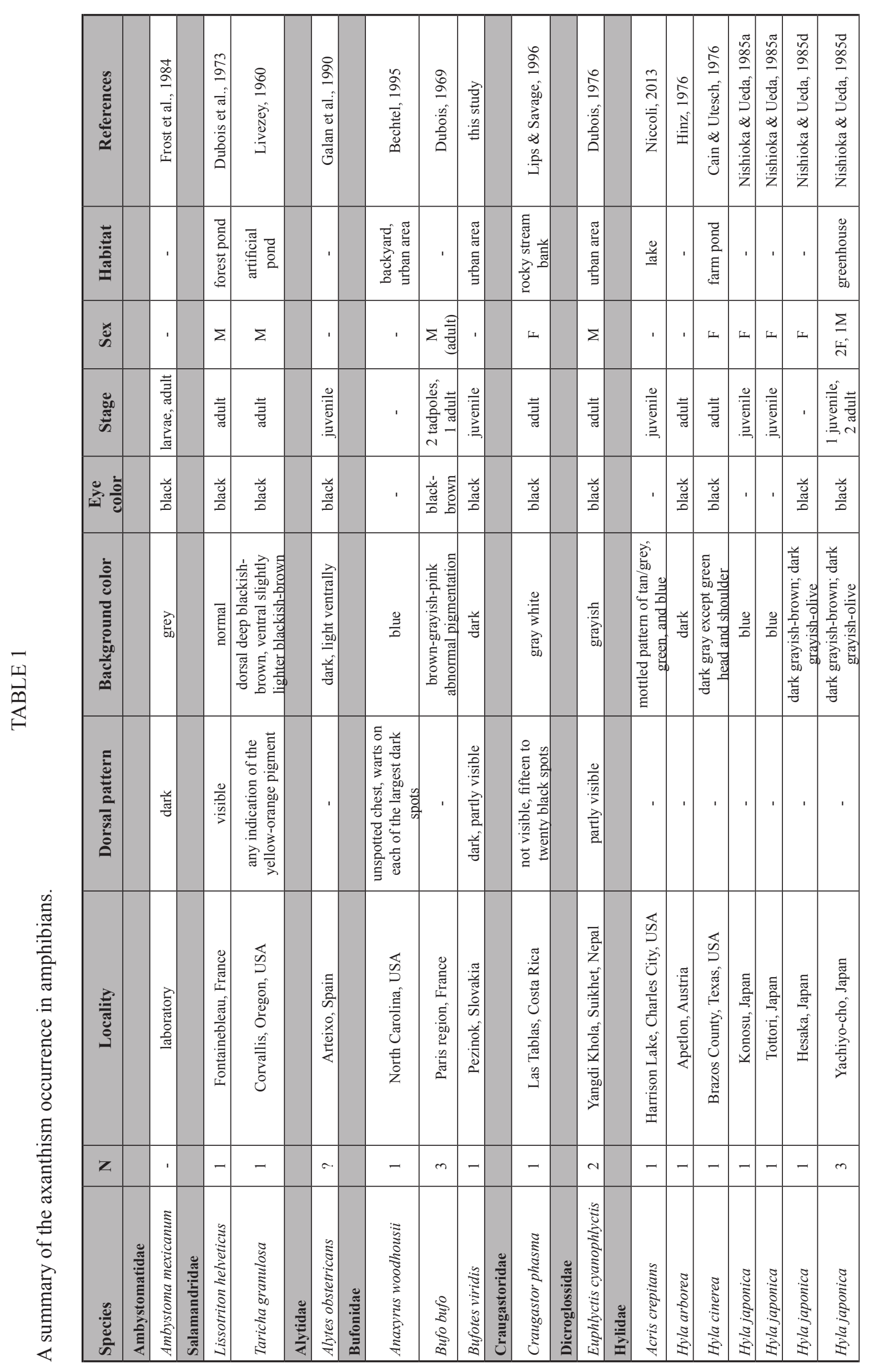




\begin{tabular}{|c|c|c|c|c|c|c|c|c|c|c|c|c|c|c|c|c|c|c|c|c|c|c|c|}
\hline & 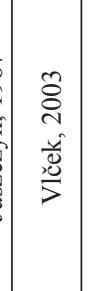 & 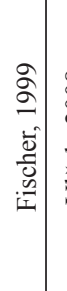 & 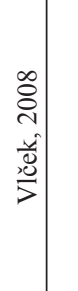 & 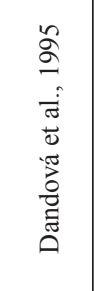 & 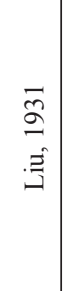 & 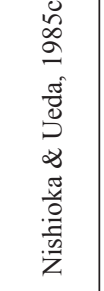 & 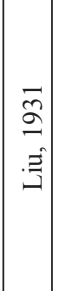 & 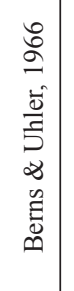 & 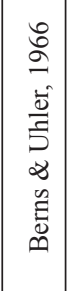 & 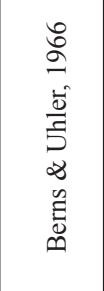 & 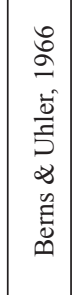 & 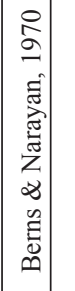 & 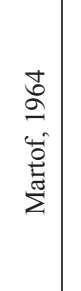 & 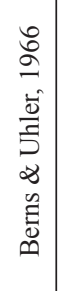 & 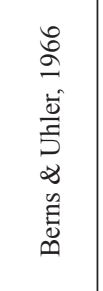 & 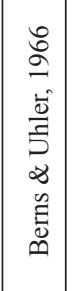 & $\begin{array}{l}\hat{\swarrow} \\
ٌ \\
\hat{u} \\
\frac{\tilde{u}}{\infty}\end{array}$ & 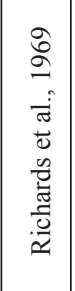 & 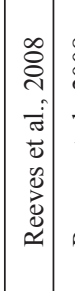 & 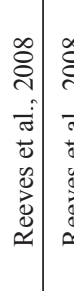 & 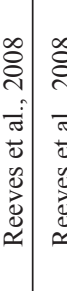 & 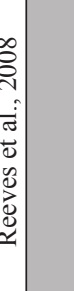 & 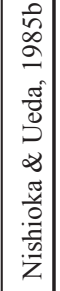 \\
\hline & 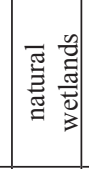 & 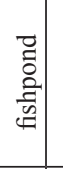 & 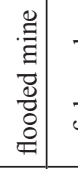 & 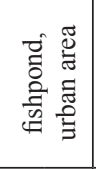 & $\begin{array}{l}\vec{z} \\
\overline{0} \\
0 \\
\underline{0} \\
\underline{0} \\
\underline{0}\end{array}$ & ' & $\begin{array}{l}\bar{z} \\
\bar{a} \\
0 \\
0 \\
0 \\
0\end{array}$ & & ' & ' & . & . & ' & & ' & I & ' & ' & 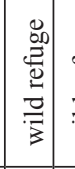 & 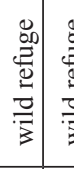 & 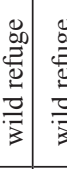 & & 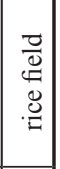 \\
\hline & $\Sigma$ & 工 & $\Sigma$ & $\Sigma$ & ' & L & ' & ' & ' & ' & ' & ' & I & & ' & ' & ' & ' & ' & ' & & & 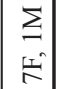 \\
\hline 壹 & $\frac{\vec{J}}{\bar{z}}$ & $\begin{array}{l}\frac{n}{3} \\
\bar{z} \\
\sigma\end{array}$ & $\frac{\vec{n}}{\vec{\Xi}}$ & 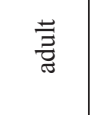 & ' & 奇 & 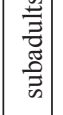 & ' & ' & ' & ' & ' & 䔍 & ' & ' & ' & ' & 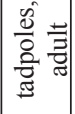 & ' & ' & & & $\frac{ \pm}{\frac{\vec{z}}{\sigma}}$ \\
\hline $\begin{array}{l}\frac{u}{\tilde{g}} \\
\frac{\pi}{\partial}\end{array}$ & $\begin{array}{l}\frac{\ddot{u}}{\tilde{g}} \\
\frac{\pi}{3}\end{array}$ & $\begin{array}{l}\frac{4}{0} \\
\frac{\tilde{m}}{b}\end{array}$ & $\begin{array}{l}\frac{u}{\underline{m}} \\
\frac{\tilde{m}}{0}\end{array}$ & $\begin{array}{l}\frac{\ddot{0}}{0} \\
\frac{\pi}{0}\end{array}$ & ' & 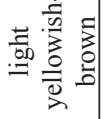 & & ' & & ' & ' & 1 & & & & & I & $\begin{array}{l}\frac{\ddot{0}}{0} \\
\frac{\pi}{0}\end{array}$ & 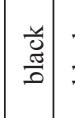 & \begin{tabular}{l|l}
$\frac{4}{0}$ \\
$\frac{\pi}{0}$
\end{tabular} & 竞 & | & $\begin{array}{l}\frac{4}{0} \\
\frac{\pi}{\partial}\end{array}$ \\
\hline & 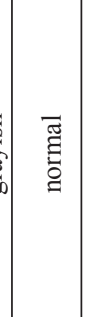 & 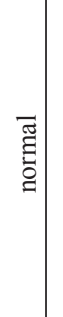 & 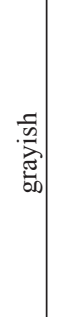 & 憘 & $\stackrel{\varrho}{g}$ & 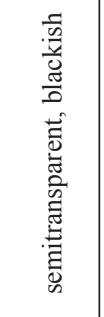 & $\frac{\varrho}{\partial}$ & 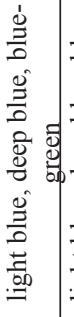 & 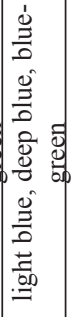 & 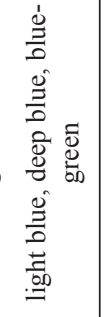 & 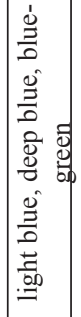 & 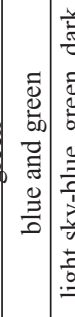 & 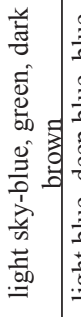 & 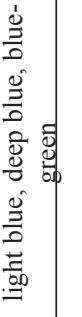 & 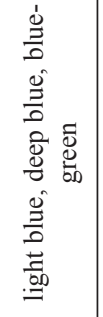 & 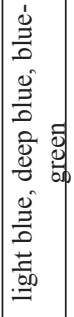 & 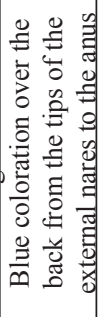 & 产 & ' & ' & & & 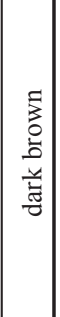 \\
\hline 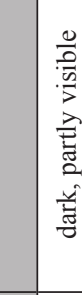 & 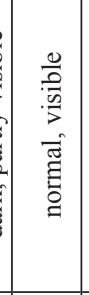 & \begin{tabular}{l|}
$\frac{0}{0}$ \\
$\frac{0}{2}$ \\
$\frac{5}{2}$ \\
$\frac{5}{5}$ \\
$\frac{5}{4}$
\end{tabular} & 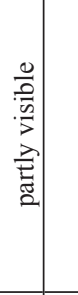 & 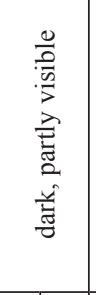 & ' & 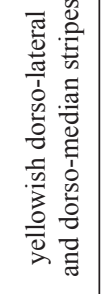 & & ' & ' & ' & ' & ' & 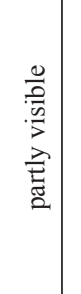 & & ' & ' & 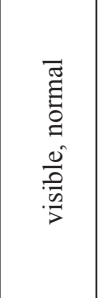 & 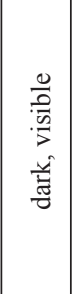 & ' & ' & & & \\
\hline $\begin{array}{l}\text { ज्ञ } \\
\text { 흉 }\end{array}$ & 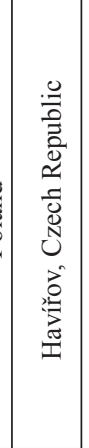 & 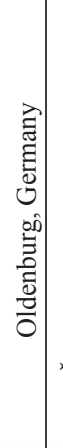 & 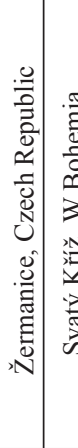 & 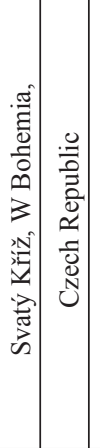 & 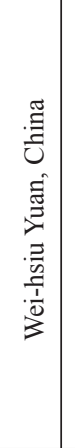 & 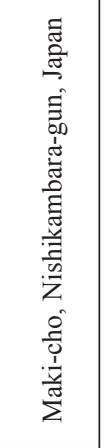 & 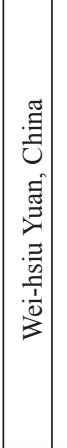 & 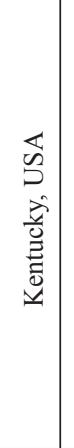 & 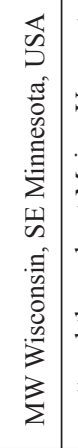 & 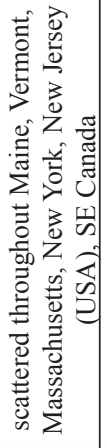 & 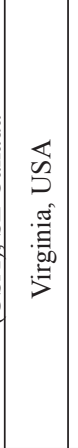 & 岕 & 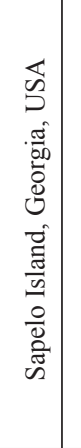 & 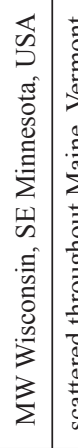 & 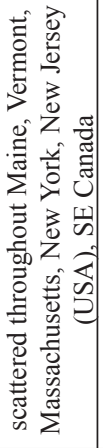 & 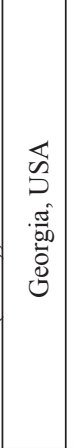 & 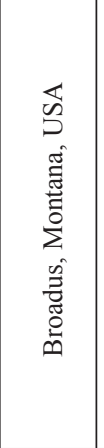 & 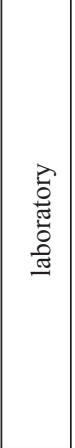 & 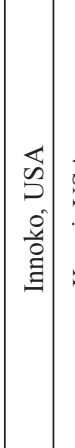 & 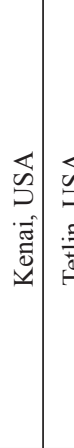 & 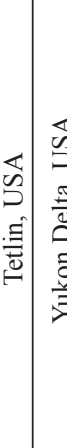 & 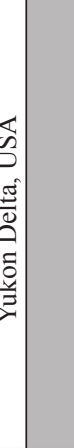 & 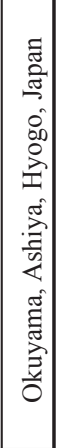 \\
\hline- & - & - & - & - & - & - & - & - & 8 & $\bar{m}$ & -1 & $\mathrm{~N}$ & - & $m$ & 0 & - & - & ' & $\because$ & $\stackrel{\infty}{=}$ & ते - & & $\infty$ \\
\hline 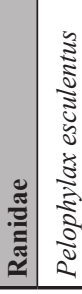 & 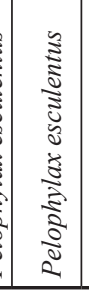 & 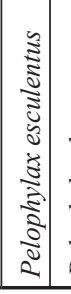 & 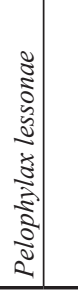 & 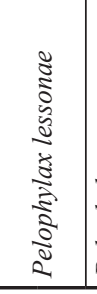 & 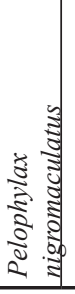 & 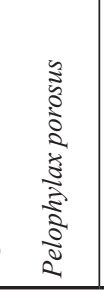 & 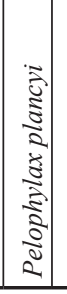 & 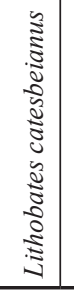 & 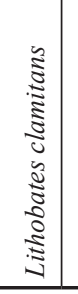 & 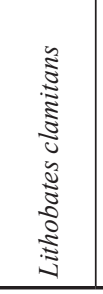 & 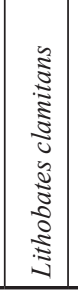 & 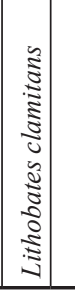 & 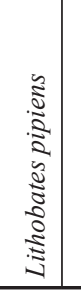 & 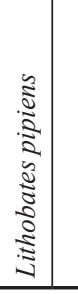 & 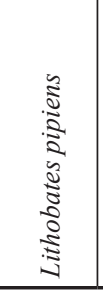 & 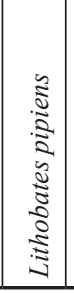 & 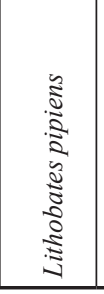 & 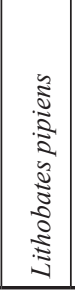 & 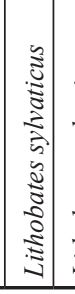 & 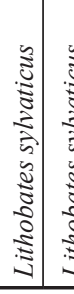 & 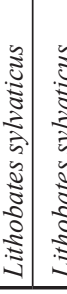 & 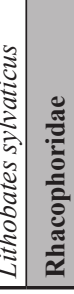 & 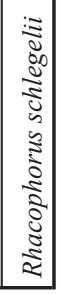 \\
\hline
\end{tabular}


the lack of certain types of chromatophores and harmful mutations, decreasing the chance of individual survival and/or genetic fixation of this aberration at the population level. All of this can underlie the relative rarity of this aberration in general and in adult specimens in particular (DuBOIS, 1979).

\section{TAXONOMIC DISTRIBUTION OF AXANTHISM IN AMPHIBIANS AND FREQUENCY OF OCCURRENCE}

Despite relatively broad taxonomic distribution of axanthism in amphibians (9 families, 23 species of the Americas, Asia and Europe, see Table 1), we can consider it a rare aberration. The largest number of examples was reported from the family Ranidae (23 cases from 9 species; cf. BERNS \& UHLER, 1966; DUBOIS \& VACHARD, 1971; DUBOIS, 1979) and it seems to be far more common in frogs than in any other group of amphibians (see Table 1). The data on intraspecific or intrapopulation frequencies are scarce and difficult to obtain. Berns and Uhler (1966) reported frequencies within different localities, finding two blue specimens out of 1000 normal frogs $(0.2 \%)$ and 22 out of $7000(0.3 \%)$ frogs for Lithobates clamitans. However, Dubois (1979) reported significantly higher frequencies $101(8.5 \%)$ of blue post-metamorphic specimens in 1186 Pelophylax sp. from France and four (3.2\%) among 126 specimens from Iran, while the same author found only eight $(0.2 \%)$ blackeyed frogs among 4651 Pelophylax sp. and one (0.1\%) in 777 Bufo bufo (DuBoIs, 1969; Dubois, 1979).

Data on frequencies of other color aberrations occurring in amphibians (albinism, leucism, melanism etc.) are scattered or completely lacking. The literature record of albinism and leucism is extensive (see e.g. WERNER, 1893; SMALlCOMBE, 1949; BRAME, 1962; CAMPANNA, 1973; DUBOIS, 1979; DYRKACZ, 1981; BECHTEL, 1995; MiKulíčEK et al., 2001; MitCHELL, 2002; PABIJAN ET AL., 2004; SPADOLA \& INSACCO, 2010; LÓPEZ \& GHIRARDI, 2011; MODESTI et al., 2011; TOLEDO et al., 2011; ESCORIZA, 2012; KEELY \& MALDONADO, 2013 and references therein), so it can be easily assumed that these two aberrations are more frequent than axanthism in natural populations. On the other hand, albinotic or leucistic specimens are more conspicuous and more interesting to record, while axanthic specimens could be misidentified as melanic or entirely overlooked, so this comparison should be treated with care. Contrary to albinism and leucism, the frequency of melanism and piebaldism seems to be lower than that of axanthism in amphibians (cf. BECHTEL, 1995).

Axanthism represents an interesting color aberration of amphibians that deserves more attention. So far, it is only logical that most cases of its occurrence are known from countries where most of the herpetological research has been carried out, i.e. North America and Europe. With more studies appearing from the other parts of the world, we can expect that both taxonomic and geographical distribution of axanthism will expand.

\section{ACKNOWLEDGMENTS}

We wish to thank Peter Mikulíček for his kind comments on the first draft of the manuscript, the terrific Tyler Square for language check, and two anonymous reviewers for their critical suggestions.

\section{REFERENCES}

ANDRÄ E (2011). Aspekte der Biologie der Wechselkröte. Wechselkröten-Symposium des LBV am 13. Mai 2011, München.

ANDRÉN C \& NilSON G (1981). Reproductive success and risk of predation in normal and melanistic colour morphs of the adder, Vipera berus. Biological Journal of the Linnean Society, 15:235-246.

ARNOLD EN \& OVENDEN D (2002). A field guide to the reptiles and amphibians of Britain and Europe. 2nd Edition. HarperCollins Publishers, London. 
BeChtel HB (1995). Reptile and amphibian variants:colors, patterns, and scales. Krieger Publishing Company, Malabar, Florida.

BERNS MW \& NARAYAN KS (1970). An histochemical and ultrastructural analysis of the dermal chromatophores of the variant blue frog. Journal of Morphology, 132:169-180.

BERNS MW \& UHLER LD (1966). Blue frogs of the genus Rana. Herpetologica, 22:181-183.

BLACK JH (1967). A blue leopard frog from Montana. Herpetologica, 23:314-315.

BRAME AHJR (1962). A survey of albinism in salamanders. Abhandlungen und Berichte für Naturkunde und Vorgeschichte, 11:65-81.

BROWDER LW (1968). Pigmentation in Rana pipiens:I. Inheritance of the speckle mutation. Journal of Heredity, 59:163-167.

CABallero L, Benítez M, Alvarez-Buylla ER, HERNÁNDEZ S, ARZOLA AV \& COCHO G. (2012). An epigenetic model for pigment patterning based on mechanical and cellular interactions. Journal of Experimental Zoology (Molecular and Developmental Evolution), 318:209-223.

CAIN BW \& UTESCH SR (1976). An unusual color pattern of the green tree frog, Hyla cinerea. Southwestern Naturalist, 21:235-236.

CAMPANNA E (1973). Biological observation on the incomplete albinism of the Crested newt (Triturus cristatus, Laur.). Archives of Biology, 84:377415.

CHILDS HE (1953). Selection by predation on albino and normal spadefoot toads. Evolution, 7:228233.

DANDOVÁ R, KotLík P \& ZAVADIL V (1995). Ein Fall von Albinismus beim Kleinen Wasserfrosch. Salamandra (Rheinbach), 31:57-60.

DubOIS A \& VACHARD D (1971). Sur une anomalie pigmentaire de la grenouille verte Rana esculenta et de quelques autres amphibiens anoures et urodeles. Bulletin Mensuel de la Société Linnéenne de Lyon, 40:40-52.

Dubois A (1969). Sur un crapaud commun aux yeux noirs. Bulletin Mensuel de la Société Linnéenne de Lyon, 38:105-106.

Dubois A (1976). Deux Rana cyanophlyctis du Nepal aux yeux noirs (Amphibiens, Anoures). Bulletin de la Societe Linneenne de Lyon, 45:303-307.

DuboIs A (1979). Anomalies and mutations in natural populations of the Rana "esculenta" complex (Amphibia, Anura). Mitteilungen aus dem Zoologischen Museum in Berlin, 55:59-87.

Dubois A, Fisher JL \& PAYEN D (1973). Un Triton palmé (Triturus helveticus) aux yeux noirs. Comptes Rendus des Séances et Mémoires de la Société de Biologie, 167:1733-1735.

Duellman WE \& TRUeB L (1994). Biology of Amphibians. John Hopkins University Press, Baltimore.

DYRKACZ S (1981). Recent instances of albinism in North American amphibians and reptiles. SSAR, Herpetological Circular, 11:1-31.

Engelmann WE \& OBST FJ (1976). Partielle Pigmentlosigkeit bei Bufo viridis viridis (Amphibia, Anura, Bufonidae). Zoologische Abhandlungen Staatliches Museum für Tierkunde in Dresden, 34:39-41.

ESCORIZA D (2012). Description of a case of albinism in a tadpole of Discoglossus pictus pictus (Anura:Discoglossidae) in Sicily. Herpetology Notes, 5:311-312.

FISCHER C (1999). Teichfrosch (Rana kl. esculenta) mit beidäugig schwarzer iris. Salamandra (Rheinbach), 35:57-59.

FLINDT R (1985). Latenter Albinismus und Mißbildungen bei Kaulquappen von Wechselkröten Bufo viridis LAURENTI, 1768. Salamandra (Rheinbach), 21:298-303.

Frost DR(2014). Amphibian Species of the World:an Online Reference. (Internet address:http:// research.amnh.org/herpetology/amphibia/index. html).

Frost SK, BRIGGS F \& MALACINSKI GM (1984). A color atlas of pigment genes in the Mexican axolotl (Ambystoma mexicanum). Differentiation, 26:182-188.

FROST-MASON SK \& MASON KA (1996). What insights into vertebrate pigmentation has the axolotl model system provided? International Journal of Developmental Biology, 40:685-693.

Galan P, Vences M, Glaw F, Fernandez Arias G \& GARCIA-PARIS M (1990). Beobachtungen zur Biologie von Alytes obstetricans in Nordwestiberian. herpetofauna, 12:17-24.

GVOŽDíK L (1999). Colour polymorphism in a population of the common lizard, Zootoca vivipara (Squamata:Lacertida). Folia Zoologica, 48:131-136. 
HINZ W (1976). Ein weiterer melanistischer Laubfrosch (Hyla arborea). Mitteilungen der Zoologischen Gesellschaft Braunau, 2:231-232.

HOFFMAN EA \& BLOUIN MS (2000). A review of colour and pattern polymorphisms in anurans. Biological Journal of the Linnean Society, 70:633665.

JAMBRICH A \& JANDZIK D (2012). Melanism in the topotypic population of the Pannonian subspecies of the common lizard, Zootoca vivipara pannonica (Reptilia:Lacertidae). Herpetology Notes, 5:219-221.

JUSZCZYK W (1987). Płazy i gady krajowe, część 2, Plazy - Amphibia; PWN, Warszawa, Poland.

KeEly CC \& MALdonAdo SP (2013). Litoria raniformis (Growling Grass Frog). Leucism. Herpetological Review, 44:297.

LANZA B \& CANeSTRELli D (2002). Atypische Färbung bei Salamandrina terdigitata (Lacepede, 1788) und Bufo viridis viridis Laurenti, 1768. Salamandra (Rheinbach), 38:105-108.

LIPS KR \& SAVAGE JM (1996). A new species of rainfrog, Eleutherodactylus phasma (Anura:Leptodactylidae), from montane Costa Rica. Proceedings of the Biological Society of Washington, 109:744-748.

LIU C. (1931). The occurrence of blue frogs in the Peiping region. China Journal, 15:246-249.

LIVEZEY RL (1960). A melanistic Taricha granulosa granulosa Skilton. Herpetologica, 16:129.

LÓPEZ JA \& GHIRARDI R (2011). First record of albinism in Rhinella fernandezae (Gellardo, 1957). Belgian Journal of Zoology, 141:59-61.

MARTOF BS (1964). An unusual color variant of Rana pipiens. Herpetologica, 17:269-270.

MiKULÍČEK P, KAUTMAN J \& ZAVADIL V (2001). Rana ridibunda albinos from eastern Slovakia living syntopically with other central european waterfrog taxa. Acta Rerum naturalium Musei Nationalis Slovaci, 47:90-94.

MitCHELL JC (2002). Leucistic Marbled Salamanders (Ambystoma opacum) in Virginia. Banisteria, 20:67-69.

Mitchell RW, RUSSELl WH \& ELliot WR (1977). Mexian eyeless characin fishes, genus Astyanax:environment, distribution, and evolution. Special Publications the Museum Texas Tech University, Texas Tech Press, Lubbock, Texas.
Modesti A, Aguzzi S \& MANenti R (2011). A case of complete albinism in Lissotriton vulgaris meridionalis. Herpetology Notes, 4:395-396.

NeVo E (1973). Adaptive color polymorphism in cricket frogs. Evolution, 27:353-367.

NicCOLI JR (2013). Acris crepitans (Northern Cricket Frog). Axanthism. Herpetological Review, 44:117.

NishioKA M \& UEDA H (1985A). Blue variants in Hyla arborea japonica. Scientific report of the Laboratory for Amphibian Biology, Hiroshima University, 7:181-197.

NishioKA M \& UEDA H (1985B). Electronmicroscopic observation on the dermal chromatophoes of normal frogs and three kinds of color variants in Rhacophorus schlegelii. Scientific report of the Laboratory for Amphibian Biology, Hiroshima University, 7:123-155.

NishioKA M \& UEDA H (1985C). Non-inheritable color variants in Rana brevipoda porosa. Scientific report of the Laboratory for Amphibian Biology, Hiroshima University, 7:199-217.

NisHiOKA M \& UEDA H (1985D). Two kids of blackeyed variants in Hyla arborea japonica. Scientific report of the Laboratory for Amphibian Biology, Hiroshima University, 7:157-179.

PABiJan M, CZarniewsKa E \& Berger L (2004). Amelanistic phenotypes in western palearctic water frogs from Poland. Herpetozoa, 17:127134.

REEVES MK, DOPLH CL, ZiMMER H, TJEERDEMA RS \& TRUST KA (2008). Road proximity increases risk of skeletal abnormalities in wood frogs from national wildlife refuges in Alaska. Environmental Health Perspectives, 116:1009-1014.

RichaRdS CM, TARTOF DT \& NACE GW (1969). A melanoid variant in Rana pipiens. Copeia, 1969:850-852.

SCHUlZ KD (1996). Eine Monographie der Schlangengattung Elaphe Fitzinger. Bushmaster Publications, Berg, Switzerland.

SKET B \& ARNTZEN JW (1994). A black nontroglomorphic amphibian from the karst of Slovenia:Proteus anguinus parkelj n. ssp. (Urodela: Proteidae). Bijdragen tot de Dierkunde, 64:33-53.

SMALlCOMBE WA (1949). Albinism in Rana temporaria. Journal of Genetics, 49:286-290. 
SPADOLA F \& InSACCO G (2010). Incomplete albinism in Discoglossus pictus (Otth, 1837). Acta Herpetologica, 5:245-253.

STÖCK M, MORITZ C, HiCKERSON M, FrYNTA D, DujSebayeVa T, EREMCHENKo V, MACEY JR, PAPENFUSS TJ \& WAKE DB (2006). Evolution of mitochondrial relationships and biogeography of Palearctic green toads (Bufo viridis subgroup) with insights in their genomic plasticity. Molecular Phylogenetics and Evolution, 41:663-689.

StöCK M, Sicilia A, Belfiore NM, BuCKLeY D, Lo-Brutto S., Lo-Valvo M \& Arculeo M (2008). Post-Messinian evolutionary relationships across the Sicilian channel:mitochondrial and nuclear markers link a new green toad from Sicily to African relatives. BMC Evolutionary Biology, 8:56.

ThOMAs M, RotTsCheidT R \& HACHTEL M (2002). Ein Fall von Flavinismus bei Erdkröte Bufo bufo (Linnaeus, 1758) nahe Bonn. Salamandra (Rheinbach), 38:119-123.

Toledo LF, Da Silva NR \& Dos SANTos ARaúJo OG (2011). Albinism in two Amazonian frogs:Elachistocleis carvalhoi (Microhylidae) and Lithobates palmipes (Ranidae). Herpetology Notes, 4:145-146.
VERSHININ VL (2004). Frequency of iris depigmentation in urban populations of Rana arvalis Frogs. Russian Journal of Ecology, 35:5862.

VitT LJ \& CALDWELl JP (2009). Herpetology:An Introductory Biology of Amphibians and Reptiles; $3^{\text {rd }}$ Edition. Academic Press, San Diego. 697 pp.

VLČEK P (2003). Skokan zelený s černou duhovkou. ŽIVA, 51:225.

VLČEK P (2008). Axanthismus u skokana krátkonohého (Pelophylax lessonae). Herpetologické Informace, 7:15.

WERNER F (1893). Albinismus und Melanismus bei Reptilien und Amphibien. Verhandlungen der Zoologisch-Botanischen Gesellschaft in Wien, 43:4-6.

WILKENS H (1988). Evolution and genetics of epigean and cave Astyanax fasciatus (Characidae, Pisces). Evolutionary Biology, 23:271-367.

Received: 27 May 2014

Accepted: 2 September 2014

Branch editor: Dominique Adriaens 